Mantelzell-Lymphom

\title{
Rezidivtherapie mit Temsirolimus
}

\begin{abstract}
Patienten mit rezidiviertem und/oder refraktärem MantelzellLymphom (MCL) steht mit Temsirolimus eine effektive Therapieoption zur Verfügung. In der bisher größten PhaseIII-Studie zum MCL verlängerte der mTOR (mammalian Target of Rapamycin)-Inhibitor das progressionsfreie Überleben im Vergleich zur Standardchemotherapie (Hess G et al., 2009, J Clin Oncol 27: 3822-3829).
\end{abstract}

In dieser Studie wurde Temsirolimus in zwei Dosierungen (175mg/Woche für drei Wochen, gefolgt von $75 \mathrm{mg}$ oder $25 \mathrm{mg}$ wöchentlich) mit einer vom jeweiligen Zentrum gewählten Monochemotherapie verglichen, erläuterte Georg Heß, Mainz, das Studiendesign. 162 vorbehandelte Patienten mit refraktärem oder rezidiviertem MCL wurden in die Studie eingeschlossen. Die Patienten hatten bereits zwei bis sieben Vortherapien, eingeschlossen Rituximab, Anthrazykline und Alkylanzien, erhalten. Bei 32\% der Patienten war bereits eine Stammzelltherapie durchgeführt worden.

\section{Reduziertes Progressionsrisiko}

Es zeigten sich deutliche Vorteile zugunsten der Therapie mit Temsirolimus: Während auf die konventionelle Chemotherapie nur 2\% der Patienten ansprachen, lag die Ansprechrate mit Temsirolimus (175/75mg) bei $22 \%(p=0,0019)$. Die gute Wirksamkeit von Temsirolimus zeigte sich zudem in einer statistisch und klinisch signifikanten Verbesserung des progressionsfreien Überlebens als primärem Endpunkt: Die mit konventioneller Chemotherapie behandelten Patienten lebten median nur 1,9 Monate ohne Progression, die mit Temsirolimus (175/75 mg) behandelten Patienten dagegen median 4,8 Monate ( $p=0,0009)$. Das Gesamtüberleben wurde durch die höher dosierte Temsirolimus-Therapie tendenziell verbessert und stieg von median 9,7 Monaten mit konventioneller Chemotherapie auf median 12,8 Monate ( $p=0,3519$; Hazard-Ratio 0,80; 95-\%-Konfidenzintervall 0,50-1,28). Die fehlende Signifikanz im Gesamtüberleben ist laut Heß auf die kleine Patientenzahl rund 55 Patienten pro Studienarm - zurückzuführen. $K A$

Quelle: Veranstaltung der Wyeth Pharma GmbH, ein Unternehmen der Pfizer-Gruppe

\section{Endokrine Therapie des Mammakarzinoms}

\section{Compliance - großer Handlungsbedarf}

\begin{abstract}
Die adjuvante endokrine Therapie ist eine effektive Behandlungsoption bei Patientinnen mit Hormonrezeptor-positivem Mammakarzinom. Die Compliance der Patientinnen ist für den Therapieerfolg dieser Langzeittherapie wichtig. Sie hängt u.a. von der Verträglichkeit und dem Nebenwirkungsmanagement ab.
\end{abstract}

Die Switchtherapie mit Exemestan (zwei bis drei Jahre Tamoxifen, anschließend Exemestan bis zum Ende der fünfjährigen

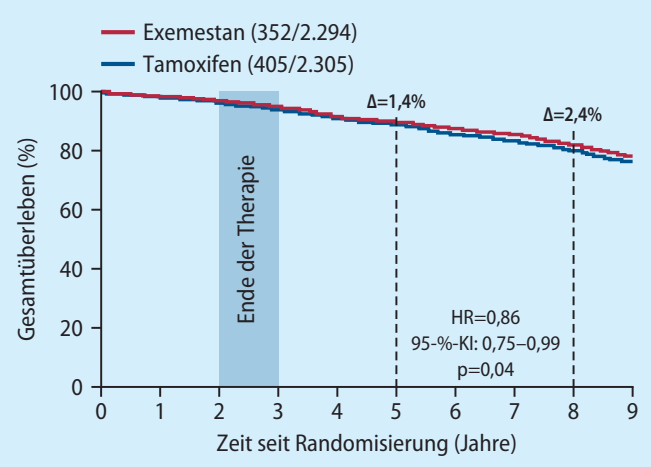

95-\%-KI=95-\%-Konfidenzintervall; HR=Hazard-Ratio

nach Bliss JM et al., 2009, SABCS: \#12, oral presentation

IES-Studie - Gesamtüberleben postmenopausaler Mammakarzinom-Patientinnen mit positivem/ unbekanntem Hormonrezeptor-Status:

Switchtherapie mit Exemestan versus Tamoxifen endokrinen Therapie) kann nicht nur das krankheitsfreie Überleben (DFS) im Vergleich zu Tamoxifen verbessern, sondern auch das Gesamtüberleben (OS) verlängern (Coombes RC et al., 2007, Lancet 369: 559570). Wie Marc Sütterlin, Mannheim, betonte, setzt sich dieser Effekt in der IES (Intergroup Exemestane Study) nach Behandlungsende fort bzw. vergrößert sich sogar (Abb.; Bliss JM et al., 2009, SABCS: \#12, oral presentation). Der Switch auf einen Aromatasehemmer (Al) nach zwei bis drei Jahren Tamoxifen wurde erneut von der Arbeitsgemeinschaft Gynäkologische Onkologie (AGO) mit dem höchsten Empfehlungsgrad ausgezeichnet (www.ago-online.de).

Das beste Arzneimittel wirkt jedoch nur, wenn es genommen wird, so Sütterlin. In dieser Hinsicht gibt es erheblichen Handlungsbedarf, wie die Ergebnisse einer großen Studie (Chan A et al., 2009, SABCS: \#36) implizieren, in der etwa $40 \%$ der Pati- entinnen mit adjuvanter endokriner Therapie non-compliant waren.

Zu den wichtigsten Faktoren, die die Compliance beeinflussen, gehören $\mathrm{Ne}$ benwirkungen, wie vor allem die Al-assoziierten Arthralgien. Leider gibt es keine direkten Vergleichsstudien, so Sütterlin. Die Angaben in den Fachinformationen legten aber den Schluss nahe, dass das Arthralgie-Risiko unter Exemestan geringer ist. Für Exemestan wird dort eine Arthralgie-Rate von $18 \%$ angegeben, für die nichtsteroidalen Al Anastrozol und Letrozol eine Rate von 35,6\% bzw. 28,5\% (Fachinformation Aromasin $^{\oplus}$ : Stand Mai 2009; Fachinformation Arimidex ${ }^{\circledast}$ : Stand Dezember 2009; Fachinformation Femara ${ }^{\circledast}$ : Stand Juni 2009). Der Wechsel auf Exemestan kann bei Patientinnen, bei denen infolge eines nichtsteroidalen Al Arthralgien auftreten, zu einer Linderung der Beschwerden führen. GS Quelle: Veranstaltung der Pfizer Pharma GmbH 